\title{
Nomogram to predict pathologic complete response in HER2-positive breast cancer treated with neoadjuvant systemic therapy
}

\begin{abstract}
Takeo Fujii ${ }^{1,2}$, Takahiro Kogawa ${ }^{1}$, Jimin $\mathrm{Wu}^{3}$, Aysegul A Sahin ${ }^{4}$, Dian D Liư ${ }^{3}$, Mariana Chavez-MacGregor ${ }^{1,5}$, Sharon H Giordano ${ }^{1}$, Akshara Raghavendra ${ }^{1}$, Rushmy K Murthy ${ }^{1}$, Debu Tripathy ${ }^{1}$, Yu Shen ${ }^{3}$, Jose-Miguel Yamal ${ }^{2}$ and Naoto T Ueno*,1

${ }^{1}$ Department of Breast Medical Oncology, The University of Texas MD Anderson Cancer Center, 1515 Holcombe Boulevard, Houston, Texas 77030, USA; ${ }^{2}$ Department of Biostatistics, The University of Texas Health Science Center at Houston School of Public Health, 1200 Pressler Street, Houston, Texas 77030, USA; ${ }^{3}$ Department of Biostatistics, The University of Texas MD Anderson Cancer Center, 1515 Holcombe Boulevard, Houston, Texas 77030, USA; ${ }^{4}$ Department of Pathology, The University of Texas MD Anderson Cancer Center, 1515 Holcombe Boulevard, Houston, Texas 77030, USA and ${ }^{5}$ Department of Health Services Research, Division of Cancer Prevention, The University of Texas MD Anderson Cancer Center, 1515 Holcombe Boulevard, Houston, Texas 77030, USA
\end{abstract}

Background: Pathologic complete response ( $P C R$ ) is associated with improved survival outcomes in patients with HER2-positive primary breast cancer. We developed a nomogram to predict the probability of $\mathrm{pCR}$ rates by using oestrogen receptor (ER) expression, progesterone receptor (PR) expression and HER2/CEP17 ratio as continuous variables.

Methods: We retrospectively reviewed patients with stages I-III HER2-positive invasive breast cancer who had definitive surgery in 1999-2015 and received neoadjuvant systemic therapy (NST). Multivariate logistic regression models were applied to assess the effect of variables on PCR. A nomogram was built to estimate the probability of pCR. The discriminative ability was estimated by the concordance index (C-index). The accuracy was assessed graphically with a calibration curve.

Results: A total of 793 patients were included in the analysis. Low ER expression $(P<0.001)$, high HER2/CEP12 ratio $(P=0.03)$, and non-inflammatory breast cancer subtype $(P=0.003)$ were associated with increased $p C R$ rates. Regimens containing trastuzumab or trastuzumab and pertuzumab were associated with higher $\mathrm{PCR}$ rates than cytotoxic agents alone $(P<0.001$ and $P<0.001$, respectively). The $\mathrm{C}$-index was 0.69 . The calibration curve showed good agreement.

Conclusions: Our nomogram predicted the pCR rate after NST among patients with HER2-positive primary breast cancer using clinicopathologic factors.

In the United States, breast cancer is the most common newly diagnosed cancer and the second most common cause of cancerrelated death in women (Siegel et al, 2015). Human epidermal growth factor receptor 2 (HER2) is overexpressed in $\sim 20 \%$ of patients with primary invasive breast cancer. HER2 amplification or overexpression is associated with an aggressive tumour phenotype, and patients with HER2-positive breast cancer had shorter survival durations than those with normal HER2 expression until the monoclonal antibody for HER2, trastuzumab (TmAb) (Herceptin, Genentech Inc., South San Francisco, CA, USA), was developed (Slamon et al, 1987, 1989; Press et al, 1997).

Neoadjuvant systemic therapy (NST) in combination with antiHER2-targeted therapy has been well established as a treatment strategy for HER2-positive breast cancer. Patients with HER2-

*Correspondence: Professor NT Ueno; E-mail: nueno@mdanderson.org

Received 8 August 2016; revised 13 November 2016; accepted 14 December 2016; published online 12 January 2017

(c) 2017 Cancer Research UK. All rights reserved 0007-0920/17 
positive breast cancer obtain additional clinical benefit from NST when it is combined with TmAb; higher pathologic complete response ( $\mathrm{pCR}$ ) rates and better survival outcomes are achieved in these patients compared with those treated with cytotoxic agents alone (Gianni et al, 2010). Moreover, anti-HER2 doublet therapy combined with cytotoxic agents has recently become a standard NST regimen for HER2-positive breast cancer. Neoadjuvant chemotherapy combined with TmAb plus lapatinib demonstrated improved pCR rates compared with chemotherapy combined with single-agent TmAb (Baselga et al, 2012). More recently, prospective randomised controlled trials (NeoSphere and TRYPHAENA trials) have reported that anti-HER2 doublet therapy of TmAb and pertuzumab yielded higher pCR rates and better survival outcomes than single-agent anti-HER2-targeted therapy (Gianni et al, 2012; Schneeweiss et al, 2013). Currently, we recommend the anti-HER2 doublet therapy of $\mathrm{TmAb}$ and pertuzumab as a treatment in neoadjuvant setting in HER2 + breast cancer.

Sensitivity to systemic therapy is known to vary depending on hormone receptor (HR) status among patients with HER2-positive breast cancer. Previous studies reported that HR-positive tumours were less sensitive to combination systemic therapies. The pCR rate in patients with HR-positive and HER2-positive disease was 20\%, compared with $36 \%$ in those with HR-negative disease (Gianni et al, 2012). Another study showed that HR-negative status significantly increased the duration of event-free survival in patients with HER2-positive disease (hazard ratio 0.46, 95\% confidence interval (CI) $0.27-0.80$ ), but HR-positive status did not affect event-free survival (hazard ratio $0.87,95 \%$ CI $0.43-1.74$ ) (Gianni et al, 2010). pCR rates after NST with regimens that include TmAb tend to be high in patients with HR-negative and HER2-positive breast cancer. However, the optimal way of predicting which patients in this group are most likely to achieve pCR after NST with regimens that include anti-HER2-targeted therapies is still unknown. In addition, oestrogen receptor (ER) expression is currently considered as a categorical variable in clinical settings; it is unclear whether ER expression level as a continuous variable is associated with pCR.

Recently, our group showed that a high HER2/CEP17 ratio is a predictor of high pCR rates among patients with HER2-positive locally advanced breast cancer who received NST that included TmAb (Kogawa et al, 2016). Given that HR status tends to predict pCR rates, considering ER expression (as a continuous variable) together with HER2/CEP17 ratio may provide a better prediction of $\mathrm{pCR}$ rates than either variable alone. We hypothesised that high HER2/CEP17 ratios and low ER expression levels are associated with high pCR rates in patients with HER2-positive breast cancer treated with NST. We constructed a nomogram to predict pCR rates in patients with primary HER2-positive breast cancer treated with NST.

\section{MATERIALS AND METHODS}

Study design. This retrospective single-centre study was approved by the Institutional Review Board at The University of Texas MD Anderson Cancer Center (protocol number: PA 12-1173) and the University of Texas Health Science Center at Houston School of Public Health (protocol number: HSC-SPH-15-0038). Patients were identified from the Breast Cancer Management System database (protocol number: 2004-0541) at MD Anderson. We retrospectively reviewed patients with stages I-III HER2-positive invasive breast cancer who had definitive surgery in 1999-2015 and received NST. HER2-positive disease was defined as a HER2/ CEP17 ratio of $\geqslant 2.0$ according to fluorescence in situ hybridisation (FISH) or an immunohistochemistry (IHC) staining score of 3 or higher (Wolff et al, 2013). Patients for whom continuous ER and PR levels or HER2/CEP17 ratios were not available were excluded from our analysis.

We collected the following information from patient charts and the database: age, patient-reported race, menopausal status, body mass index (BMI), ER and progesterone receptor (PR) status (as a continuous variable from 0 to $100 \%)$, HER2/CEP17 ratio, histologic subtype and nuclear grade (determined from a biopsy specimen), clinical stage inflammatory breast cancer (IBC) or nonIBC, NST regimens, and treatment response (pCR or non-pCR). A waiver of informed consent was authorised because the study was a retrospective chart review involving no additional diagnostic or therapeutic intervention.

Pathologic evaluation. Diagnostic biopsy and resected specimens were evaluated by a dedicated breast pathologist at MD Anderson. ER and PR expression were evaluated in the diagnostic biopsy samples using IHC staining; the percentage of expression, ranging from 0 to $100 \%$, was calculated at the time of diagnosis. pCR after NST was defined as no evidence of residual invasive cancer in the breast and no residual cancer in the axilla at the time of definitive surgery.

Statistical analysis. The primary endpoint of interest was pCR. Variables of interest included age, race, BMI prior to NST, menopausal status, histologic findings, clinical stage, IBC or nonIBC, ER expression, PR expression, HER2/CEP17 ratio, and NST regimen (containing TmAb, TmAb plus pertuzumab (PmAb), or cytotoxic agents only). Data were summarised using descriptive statistics. Associations between categorical variables were examined using the $\chi^{2}$ test or Fisher exact test when appropriate. The Wilcoxon rank-sum test or Kruskal-Wallis test was used to examine differences in continuous variables between or among patient groups. Multivariate logistic regression models were applied to assess the effect of variables of interest on pCR status. Backward stepwise selection was applied to determine which variables were included in the final multivariable model. A nomogram was built to estimate the probability of $\mathrm{pCR}$, given the risk factors in the final multivariable model. A bootstrap validation method based on 200 bootstrap samples was employed to estimate the bias-corrected or overfitting-corrected predictive discriminative ability of the model, which was presented as the concordance index (C-index; Efron and Gong, 1983). The accuracy of the logistic regression model was assessed graphically with a calibration curve by plotting the predicted probabilities of pCR on the $x$ axis with the observed probabilities of pCR on the $y$ axis. Two-sided $P<0.05$ was considered statistically significant in all statistical analyses. All computations were carried out in SAS 9.3 (SAS Institute Inc., Cary, NC, USA) and R 3.1.2.

\section{RESULTS}

Patient characteristics. A total of 793 patients were included in the analysis, and 345 of them (43.5\%) achieved pCR. Demographic and clinicopathologic characteristics are summarised in Table 1. Four hundred ten patients $(51.7 \%)$ were postmenopausal, and postmenopausal women had significantly higher pCR rates than premenopausal women $(P=0.03)$. The mean ER expression level was $47.1 \%$ in the non-pCR group and $28.2 \%$ in the pCR group $(P<0.001)$, and the mean PR expression level was $26.2 \%$ in the non-pCR group and $14.6 \%$ in the pCR group $(P<0.001)$. Patients with non-IBC had a higher pCR rate $(44.5 \%)$ than those with IBC (34.2\%; $P=0.09$ ), although not statistically significant. Patients who received NST regimens containing TmAb or TmAb plus PmAb had significantly higher pCR rates than those receiving NST regimens containing cytotoxic agents alone (TmAb: 277 out of 596 patients, 46.5\%; TmAb plus PmAb: 42 out of 81 patients, $51.9 \%$; cytotoxic agents alone: 26 out of 116 patients, $22.4 \%$; $P<0.001$ ). 
Table 1. Baseline patient characteristics

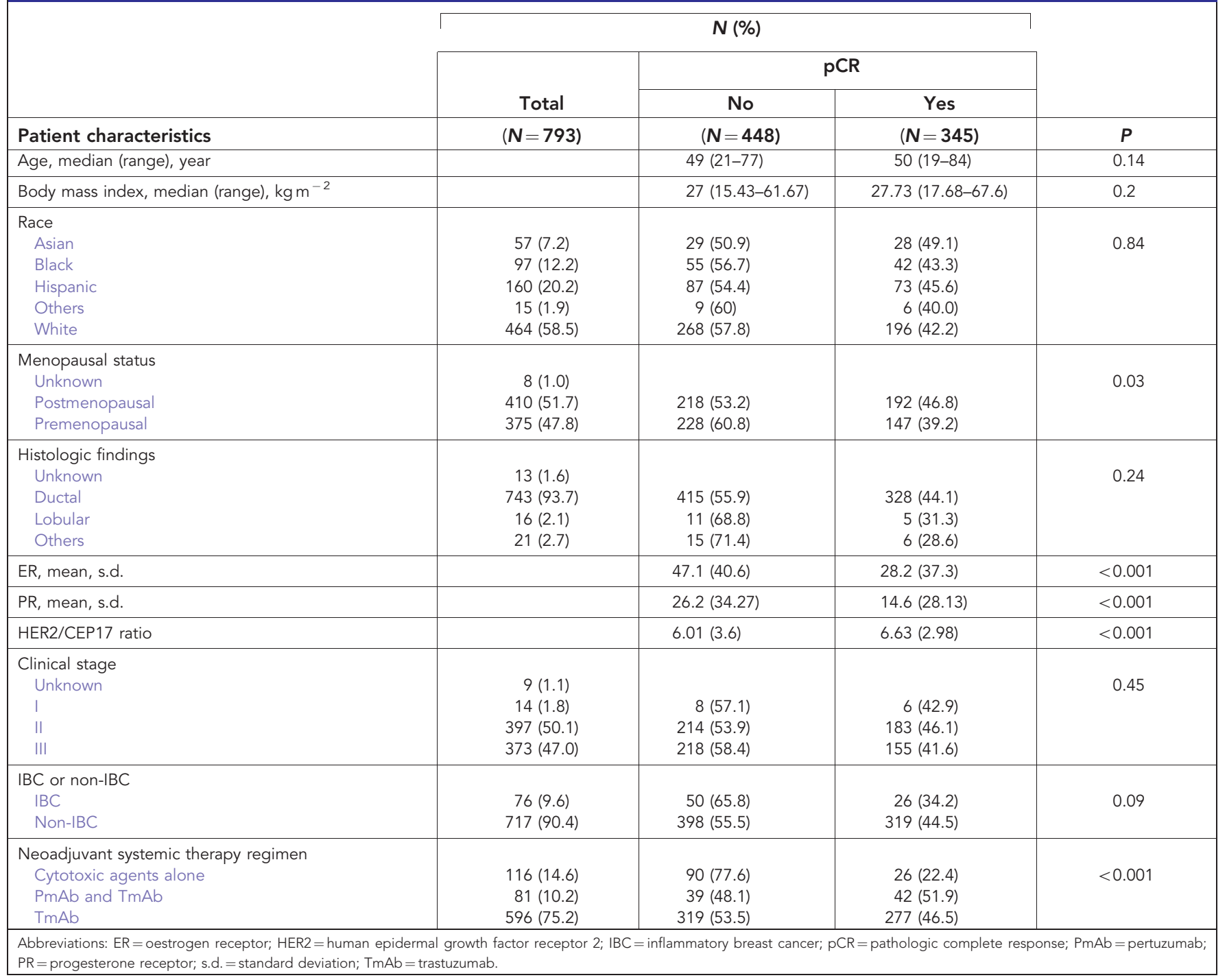

There was no significant interaction between NST regimen and HER2/CEP17 ratio by pCR rate.

Logistic regression model of $\mathrm{pCR}$ and nomogram to predict pCR. In multivariate logistic regression analysis, ER expression level, HER2/CEP17 ratio, IBC or Non-IBC, and NST regimen remained in the final model were associated with pCR rates. PR was included in the model as a variable of interest. Low ER expression was associated with higher pCR rates (odds ratio (OR) 0.99, 95\% CI 0.98-0.99, $P<0.001)$. High HER2/CEP17 ratio and non-IBC were also associated with higher pCR rates (HER2/CEP17 ratio: OR $1.05,95 \%$ CI $1.004-1.10, P=0.03$; non-IBC: OR 2.23, 95\% CI 1.30-3.82, $P=0.003$; Table 2). NST regimens containing $\mathrm{TmAb}$ and $\mathrm{TmAb}$ plus PmAb were associated with significantly higher pCR rates than NST regimens containing cytotoxic agents alone (TmAb: OR 3.41, 95\% CI 2.08-5.61, $P<0.001$; TmAb plus PmAb: OR 4.03, 95\% CI 2.05-7.91, $P<0.001$; Table 2). The final regression model of $\mathrm{pCR}$ remained similar in the analysis without IBC patients $(N=314$, Supplementary Table 1$)$. Since majority of patients with HER2 + tumour are treated with TmAb with or without $\mathrm{PmAb}$, we created a two different multivariate logistic model to see the effect of cytotoxic agents alone in the model. The first model removed cytotoxic agent alone from the model in Table 2. Even after removing cytotoxic agents, ER, PR, HER2 FISH
Table 2. Multivariate logistic regression model of pathologic complete response (345 out of 793 patients; $43.5 \%$ )

\begin{tabular}{|c|c|c|c|}
\hline Covariate & OR & $95 \% \mathrm{Cl}$ & $P$ \\
\hline ER expression & 0.99 & $0.98-0.99$ & $<0.001$ \\
\hline PR expression & 1 & $0.99-1.00$ & 0.21 \\
\hline HER2/CEP17 ratio & 1.05 & $1.004-1.10$ & 0.03 \\
\hline $\begin{array}{l}\text { IBC or non-IBC } \\
\text { IBC } \\
\text { Non-IBC }\end{array}$ & $\begin{array}{c}1 \\
2.23\end{array}$ & $1.30-3.82$ & 0.003 \\
\hline $\begin{array}{l}\text { Neoadjuvant systemic therapy regimen } \\
\text { Cytotoxic agents alone } \\
T m A b \\
T m A b \text { and } \mathrm{PmAb}\end{array}$ & $\begin{array}{c}1 \\
3.41 \\
4.03\end{array}$ & $\begin{array}{l}2.08-5.61 \\
2.05-7.91\end{array}$ & $\begin{array}{l}<0.001 \\
<0.001\end{array}$ \\
\hline
\end{tabular}

Abbreviations: $\mathrm{Cl}=$ confidence interval; $\mathrm{ER}=$ oestrogen receptor; HER2 = human epidermal growth factor receptor $2 ; \mathrm{IBC}=$ inflammatory breast cancer; $\mathrm{OR}=$ odds ratio; $\mathrm{PmAb}=$ pertuzumab; $P R=$ progesterone receptor; $\operatorname{TmAb}=$ trastuzumab.

ratio, and IBC remained same in the model and NST regimen was not significant (Supplementary Table 2). In the second model, we conducted a new model selection after removing cytotoxic agent alone, ER, PR, HER2 FISH ratio, and IBC again remained same and NST regimen was not included in the final model. (Supplementary Table 3). 


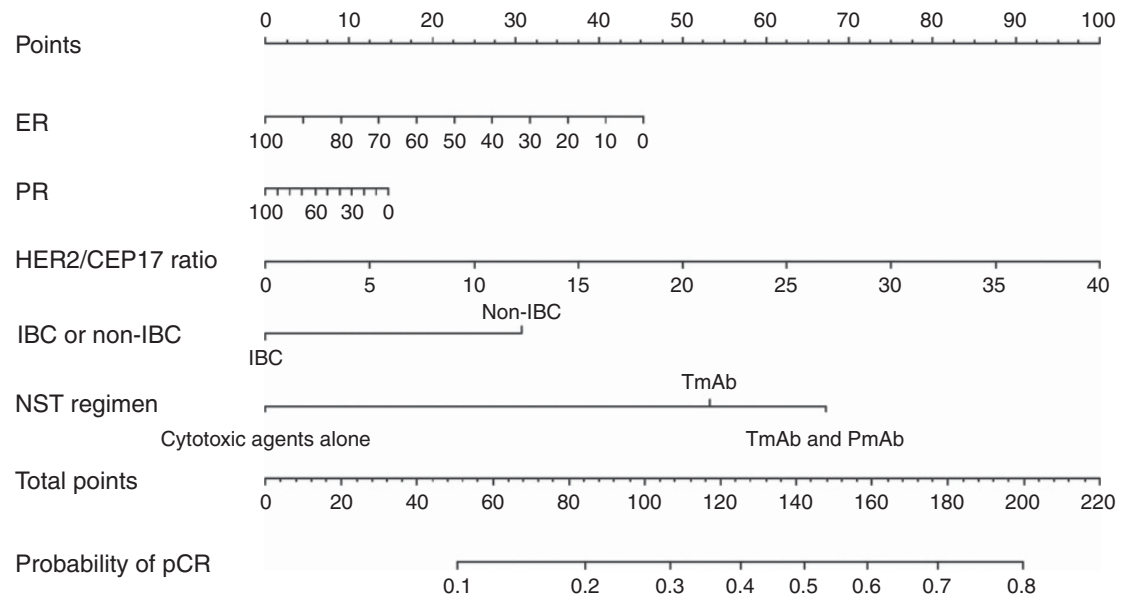

Figure 1. Nomogram to predict the probability of pathologic complete response (pCR). ER=oestrogen receptor; $I B C=$ inflammatory breast cancer; $\mathrm{PR}=$ progesterone receptor.

A nomogram constructed on the basis of the final multivariable model is shown in Figure 1. To test the discriminative ability of the model, we calculated the C-index using bootstrap methods with 200 replications. The C-index was 0.69 (95\% CI 0.66-0.73). The calibration curve used to assess the goodness-of-fit of the model (Figure 2) showed good agreement between the predicted and observed probabilities of pCR.

\section{DISCUSSION}

Our results demonstrated that high HER2/CEP17 ratios and low ER expression levels were associated with high pCR rates in patients with HER2-positive breast cancer treated with NST. On the basis of these results, we created a nomogram to predict the probability of $\mathrm{pCR}$ in patients with stages I-III HER2-positive primary breast cancer treated with NST using five covariates: ER expression level, PR expression level, HER2/CEP17 ratio, IBC or non-IBC, and NST regimen.

Previous studies have shown that the response to systemic therapy in breast cancer varies depending on the disease characteristics. Our group previously showed that a high HER2/CEP17 ratio was a significant predictor of PCR in patients with locally advanced disease who had received NST (Kogawa et al, 2016). In patients with HER2-positive breast cancer who had received NST-containing cytotoxic agents and $\mathrm{TmAb}$, negative $\mathrm{ER} / \mathrm{PR}$ status was an independent predictor of pCR in multivariate analysis (adjusted OR 3.42, 95\% CI 2.42-4.86, $P<0.001$; Takada et al, 2014). Another study demonstrated that in patients not treated with NST-containing TmAb, pCR was highest for those with HR-negative and HER2-positive disease (45\%) and lowest for those with HR-positive and HER2-negative disease (9\%) (Esserman et al, 2012). A retrospective study showed that high HER2 expression was associated with high pCR rates after treatment with the combination of lapatinib and $\operatorname{Tm} A b$, irrespective of HR status (Scaltriti et al, 2015). Some prospective studies have shown that patients with HR-negative disease were sensitive to chemotherapies, allowing them to achieve pCR more than those with HR-positive disease, which is also consistent with our results (Esserman et al, 2012; Alba et al, 2014).

The current study is, to the best of our knowledge, the first to generate a nomogram using various patient and disease characteristics to predict pCR after NST in HER2-positive breast cancer. Our study cohort also included those treated with $\mathrm{PmAb}$-containing regimens; $\mathrm{PmAb}$ was recently (September

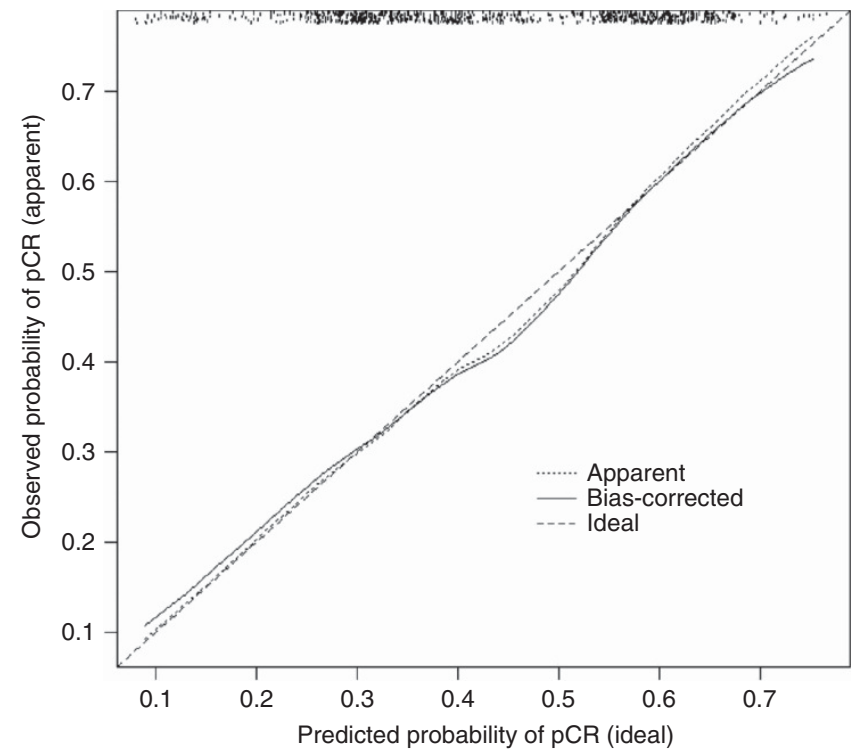

Figure 2. Calibration curve of observed and predicted probabilities. The $x$ axis is the predicted probabilities measured by the final logistic regression model and the $y$ axis is the actual probabilities.

$\mathrm{pCR}=$ pathologic complete response.

2013) approved by the US Food and Drug Administration for neoadjuvant treatment of HER2-positive breast cancer and has been the standard of care since then. In addition, in our model, ER, $\mathrm{PR}$, and HER2/CEP17 ratios were treated as continuous variables. This nomogram can provide the estimated probability of achieving pCR after the planned NST using clinicopathologic features.

Several limitations to our findings should be noted. First, this is a retrospective study and thus we could not account for unknown factors related to pCR. Second, we could not assess this nomogram in terms of survival outcomes because of the short duration of follow-up in patients in the cohort who received NST with PmAb. This is mainly because PmAb was just approved in 2013 and we did not have enough events to analyse survival outcomes based on the predicted probability obtained from the nomogram. Third, the quality of supportive care could have varied because of the long duration of the inclusion period. In particular, those who received NST-containing cytotoxic agents alone might have been treated longer ago than those who received anti-HER2-targeted therapy. In addition, relatively low-quality supportive care may have led to 
delay or discontinuation of the systemic therapy, leading to low pCR rates in the group receiving NST-containing cytotoxic agents alone. However, all patients were treated at MD Anderson and any differences in quality of the care are therefore likely to be small.

Another issue is the reproducibility of ER/PR expression level. Because we treated ER and PR as continuous variables, the percentage might change depending on the pathologist who reviewed the slides. We included clinical stage in the analysis instead of $\mathrm{T}$ stage and $\mathrm{N}$ stage separately. In ER-positive breast cancer in particular, node status can be an important indicator of survival outcomes (Sparano et al, 2015). However, our primary endpoint in the current study was pCR. In addition, all patients in our cohort had HER2-positive breast cancer and hormone status was one of the variables used in the analysis. In the current study, tumour size and node status were not assessed independently. However, the effect of it was likely small and tumour size and nodal status were included in staging that was included in analysis. Also neoadjuvant systemic treatment strategy is the same regardless of tumour size or nodal stage.

Lastly, although we used a bootstrap method to validate the model internally, we did not have an independent cohort outside of $\mathrm{MD}$ Anderson to validate the nomogram externally. Without external validation, whether the reported nomogram can be generalised in other patient cohorts such as different patient demographic characteristics and treatment outside of MD Anderson is not clear. Also the causality of the variables are still not proved. We will continue to seek such data sets for future validation study.

The C-index of our model was 0.69 . This means that our model can distinguish a patient with $\mathrm{pCR}$ from a patient without $\mathrm{pCR}$ $69 \%$ of the time. C-index values range from 0.5 to 1 ; a value of 0.5 means the discrimination is just random chance and a value of 1 indicates a perfect discriminative ability. Some important variables were not available to be considered in the model. For example, we could not evaluate the heterogeneity of the HER2-positive tumours. One study demonstrated that heterogenous tumours with a HER2/CEP17 ratio of more than 2.2 in $<80 \%$ of the tumour cells were associated with poor response to $\mathrm{TmAb}$ (Mcghan et al, 2014). We could not consider intratumor heterogeneity because we performed a retrospective chart review. This may have contributed to the low discriminative ability. Other factors may also lead to treatment resistance; several studies have investigated both intrinsic resistance and acquired resistance to treatment (Gottesman, 2002; Sui et al, 2007; Colleoni et al, 2009; Takada et al, 2014). For example, one study demonstrated that the $\mathrm{ER} \alpha$ gene could increase drug resistance in breast cancer cells by activating MDR1 transcription (Shi et al, 2014).

By using the model developed in the current study, we can estimate the probability of pCR before the start of NST. However, to answer the critical clinical question of who definitely needs $\mathrm{PmAb}$ in addition to TmAb, we need to identify biological and other clinical factors to improve the predictability of pCR in future studies.

In conclusion, patients with high HER2/CEP17 ratios and low ER expression levels are most likely benefit from NST. Our nomogram predicted PCR rate after NST among patients with HER-2 positive primary breast cancer using clinicopathologic factors. To improve the predictability of this nomogram, we need to identify other clinical and molecular factors. Further studies, especially an external validation, are warranted.

\section{ACKNOWLEDGEMENTS}

Erica Goodoff of the Department of Scientific Publications at The University of Texas MD Anderson Cancer Center provided scientific editing services. This study was funded by the Morgan Welch Inflammatory Breast Cancer Research Program; a grant from the State of Texas Rare and Aggressive Breast Cancer Research Program; MD Anderson's Cancer Center Support Grant from the National Cancer Institute, CA016672, which supports the Biostatistics Shared Resource; and St Luke's Life Science Institute, Tokyo, Japan.

\section{CONFLICT OF INTEREST}

The authors declare no conflict of interest.

\section{REFERENCES}

Alba E, Albanell J, De La Haba J, Barnadas A, Calvo L, Sanchez-Rovira P, Ramos M, Rojo F, Burgues O, Carrasco E, Caballero R, Porras I, Tibau A, Camara MC, Lluch A (2014) Trastuzumab or lapatinib with standard chemotherapy for HER2-positive breast cancer: results from the GEICAM/2006-14 trial. Br J Cancer 110: 1139-1147.

Baselga J, Bradbury I, Eidtmann H, Di Cosimo S, De Azambuja E, Aura C, Gomez H, Dinh P, Fauria K, Van Dooren V, Aktan G, Goldhirsch A, Chang TW, Horvath Z, Coccia-Portugal M, Domont J, Tseng LM, Kunz G, Sohn JH, Semiglazov V, Lerzo G, Palacova M, Probachai V, Pusztai L, Untch M, Gelber RD, Piccart-Gebhart M (2012) Lapatinib with trastuzumab for HER2-positive early breast cancer (NeoALTTO): a randomised, open-label, multicentre, phase 3 trial. Lancet 379: 633-640.

Colleoni M, Bagnardi V, Rotmensz N, Gelber RD, Viale G, Pruneri G, Veronesi P, Torrisi R, Cardillo A, Montagna E, Campagnoli E, Luini A, Intra M, Galimberti V, Scarano E, Peruzzotti G, Goldhirsch A (2009) Increasing steroid hormone receptors expression defines breast cancer subtypes non responsive to preoperative chemotherapy. Breast Cancer Res Treat 116: 359-369.

Efron B, Gong G (1983) A leisurely look at the bootstrap, the jackknife, and cross-validation. Am Stat 37: 36-48.

Esserman LJ, Berry DA, Demichele A, Carey L, Davis SE, Buxton M, Hudis C, Gray JW, Perou C, Yau C, Livasy C, Krontiras H, Montgomery L, Tripathy D, Lehman C, Liu MC, Olopade OI, Rugo HS, Carpenter JT, Dressler L, Chhieng D, Singh B, Mies C, Rabban J, Chen YY, Giri D, Van 'T Veer L, Hylton N (2012) Pathologic complete response predicts recurrence-free survival more effectively by cancer subset: results from the I-SPY 1 TRIAL-CALGB 150007/150012, ACRIN 6657. J Clin Oncol 30: 3242-3249.

Gianni L, Eiermann W, Semiglazov V, Manikhas A, Lluch A, Tjulandin S, Zambetti M, Vazquez F, Byakhow M, Lichinitser M, Climent MA, Ciruelos E, Ojeda B, Mansutti M, Bozhok A, Baronio R, Feyereislova A, Barton C, Valagussa P, Baselga J (2010) Neoadjuvant chemotherapy with trastuzumab followed by adjuvant trastuzumab versus neoadjuvant chemotherapy alone, in patients with HER2-positive locally advanced breast cancer (the NOAH trial): a randomised controlled superiority trial with a parallel HER2-negative cohort. Lancet 375: 377-384.

Gianni L, Pienkowski T, Im YH, Roman L, Tseng LM, Liu MC, Lluch A, Staroslawska E, De La Haba-Rodriguez J, Im SA, Pedrini JL, Poirier B, Morandi P, Semiglazov V, Srimuninnimit V, Bianchi G, Szado T, Ratnayake J, Ross G, Valagussa P (2012) Efficacy and safety of neoadjuvant pertuzumab and trastuzumab in women with locally advanced, inflammatory, or early HER2-positive breast cancer (NeoSphere): a randomised multicentre, open-label, phase 2 trial. Lancet Oncol 13: 25-32.

Gottesman MM (2002) Mechanisms of cancer drug resistance. Annu Rev Med 53: 615-627.

Kogawa T, Fouad TM, Liu DD, Wu J, Shen Y, Masuda H, Fujii T, Chavez-Macgregor M, Alvarez RH, Hortobagyi GN, Valero V, Ueno NT (2016) High HER2/centromeric probe for chromosome 17 fluorescence in situ hybridization ratio predicts pathologic complete response and survival outcome in patients receiving neoadjuvant systemic therapy with trastuzumab for HER2-overexpressing locally advanced breast cancer. Oncologist 21: 21-27.

Mcghan LJ, Mccullough AE, Protheroe CA, Dueck AC, Lee JJ, Nunez-Nateras R, Castle EP, Gray RJ, Wasif N, Goetz MP, Hawse JR, Henry TJ, Barrett MT, Cunliffe HE, Pockaj BA (2014) Androgen receptor-positive triple negative breast cancer: a unique breast cancer subtype. Ann Surg Oncol 21: 361-367. 
Press MF, Bernstein L, Thomas PA, Meisner LF, Zhou J-Y, Ma Y, Hung G, Robinson RA, Harris C, El-Naggar A (1997) HER-2/neu gene amplification characterized by fluorescence in situ hybridization: poor prognosis in node-negative breast carcinomas. J Clin Oncol 15: 2894-2904.

Scaltriti M, Nuciforo P, Bradbury I, Sperinde J, Agbor-Tarh D, Campbell C, Chenna A, Winslow J, Serra V, Parra JL, Prudkin L, Jimenez J, Aura C, Harbeck N, Pusztai L, Ellis C, Eidtmann H, Arribas J, Cortes J, De Azambuja E, Piccart M, Baselga J (2015) High HER2 expression correlates with response to the combination of lapatinib and trastuzumab. Clin Cancer Res 21: 569-576.

Schneeweiss A, Chia S, Hickish T, Harvey V, Eniu A, Hegg R, Tausch C, Seo JH, Tsai YF, Ratnayake J, Mcnally V, Ross G, Cortes J (2013) Pertuzumab plus trastuzumab in combination with standard neoadjuvant anthracycline-containing and anthracycline-free chemotherapy regimens in patients with HER2-positive early breast cancer: a randomized phase II cardiac safety study (TRYPHAENA). Ann Oncol 24: 2278-2284.

Shi JF, Yang N, Ding HJ, Zhang JX, Hu ML, Leng Y, Han X, Sun YJ (2014) ERalpha directly activated the MDR1 transcription to increase paclitaxelresistance of ERalpha-positive breast cancer cells in vitro and in vivo. Int J Biochem Cell Biol 53: 35-45.

Siegel RL, Miller KD, Jemal A (2015) Cancer statistics, 2015. CA Cancer J Clin 65: 5-29.

Slamon DJ, Clark GM, Wong SG, Levin WJ, Ullrich A, Mcguire WL (1987) Human breast cancer: correlation of relapse and survival with amplification of the HER-2/neu oncogene. Science 235: 177-182.

Slamon DJ, Godolphin W, Jones LA, Holt JA, Wong SG, Keith DE, Levin WJ, Stuart SG, Udove J, Ullrich A (1989) Studies of the HER-2/neu protooncogene in human breast and ovarian cancer. Science 244: 707-712.

Sparano JA, Gray RJ, Makower DF, Pritchard KI, Albain KS, Hayes DF, Geyer Jr CE, Dees EC, Perez EA, Olson Jr JA, Zujewski J, Lively T,
Badve SS, Saphner TJ, Wagner LI, Whelan TJ, Ellis MJ, Paik S, Wood WC, Ravdin P, Keane MM, Gomez Moreno HL, Reddy PS, Goggins TF, Mayer IA, Brufsky AM, Toppmeyer DL, Kaklamani VG, Atkins JN, Berenberg JL, Sledge GW (2015) Prospective validation of a 21-gene expression assay in breast cancer. N Engl J Med 373: 2005-2014.

Sui M, Huang Y, Park BH, Davidson NE, Fan W (2007) Estrogen receptor alpha mediates breast cancer cell resistance to paclitaxel through inhibition of apoptotic cell death. Cancer Res 67: 5337-5344.

Takada M, Ishiguro H, Nagai S, Ohtani S, Kawabata H, Yanagita Y, Hozumi Y, Shimizu C, Takao S, Sato N, Kosaka Y, Sagara Y, Iwata H, Ohno S, Kuroi K, Masuda N, Yamashiro H, Sugimoto M, Kondo M, Naito Y, Sasano H, Inamoto T, Morita S, Toi M (2014) Survival of HER2-positive primary breast cancer patients treated by neoadjuvant chemotherapy plus trastuzumab: a multicenter retrospective observational study (JBCRG-C03 study). Breast Cancer Res Treat 145: 143-153.

Wolff AC, Hammond ME, Hicks DG, Dowsett M, Mcshane LM, Allison KH, Allred DC, Bartlett JM, Bilous M, Fitzgibbons P, Hanna W, Jenkins RB, Mangu PB, Paik S, Perez EA, Press MF, Spears PA, Vance GH, Viale G, Hayes DF, American Society of Clinical OncologyCollege of American Pathologists (2013) Recommendations for human epidermal growth factor receptor 2 testing in breast cancer: American Society of Clinical Oncology/College of American Pathologists clinical practice guideline update. J Clin Oncol 31: 3997-4013.

This work is published under the standard license to publish agreement. After 12 months the work will become freely available and the license terms will switch to a Creative Commons AttributionNonCommercial-Share Alike 4.0 Unported License.

Supplementary Information accompanies this paper on British Journal of Cancer website (http://www.nature.com/bjc) 